\title{
Textile-based electrode for electrocardiography monitoring
}

\author{
Syaidah Md Saleh ${ }^{1}$, Nurul Ashikin Abdul-Kadir ${ }^{2}$, Fauzan Khairi Che Harun ${ }^{3}$, Dedy H. B. Wicaksono ${ }^{4}$ \\ ${ }^{1}$ School of Biomedical Engineering \& Health Sciences, Faculty of Engineering, Universiti Teknologi Malaysia, Malaysia \\ ${ }^{2,3}$ School of Electrical Engineering, Faculty of Engineering, Universiti Teknologi Malaysia, Malaysia \\ ${ }^{4}$ Department of Biomedical Engineering, Faculty of Life Sciences and Technology, Swiss German University, Indonesia
}

\section{Article Info}

Article history:

Received Dec 2, 2019

Revised Apr 8, 2020

Accepted May 15, 2020

\section{Keywords:}

Cotton fabric

Dry electrode

Electrocardiography

Graphene oxide

Reduced graphene oxide

\begin{abstract}
The increasing demand of smart garment for monitoring people's health is due to comfortability, lightweight and flexibility properties of the textile could offer to the user. The textile-based electrocardiography (ECG) electrode is an alternative of commercially available silver/silver chloride $(\mathrm{Ag} / \mathrm{AgCl})$ electrode which could cause skin allergies to certain users and is not suitable for long-term monitoring electrode. In this paper, we report the performance of reduced graphene oxide (rGO) coated cotton fabric electrode to the effect of longevity and temperature. The ECG waveform and signal-to-noise ratio (SNR) of the rGO-coated cotton electrodes were compared to that the performance of $\mathrm{Ag} / \mathrm{AgCl}$ electrodes. The reliability characterization confirmed the rGO-coated cotton fabric conductance maintain at more than $80 \%$ even after 100 days of fabrication and the conductance measurement is increasing with respect to the temperature applied. The electrode shows lower in impedance value and the performance in acquiring ECG signal is comparable with the $\mathrm{Ag} / \mathrm{AgCl}$ electrode. The vertical position rectangle-shaped electrode is recommended in measuring ECG signals. In conclusion, the rGO-coated cotton electrode with flexible dry-type electrode and excellent performance especially reliability and in capturing ECG signal had shown a promising result for further development.
\end{abstract}

This is an open access article under the CC BY-SA license.

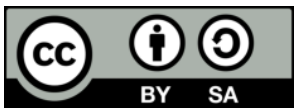

\section{Corresponding Author:}

Fauzan Khairi Che Harun,

School of Electrical Engineering,

Faculty of Engineering,

Universiti Teknologi Malaysia, 81310 UTM Johor Bahru, Johor, Malaysia.

Email: fauzan@utm.my

\section{INTRODUCTION}

The increasing health awareness among the people experienced a demand in wearable monitoring devices; to monitor individual's health, fitness, etc, in daily life. In United Kingdom, a growth of wrist-worn wearable devices like smart watches and fitness trackers during the period of year 2016 to 2017 and year 2017 to 2018 are about $18 \%$ and $7 \%$, respectively [1]. Other than wrist-worn wearable devices, research and product related to integrate light-weight sensor onto textile as a smart garment are widely explored and produced. This is due to the advent of conductive print-ink on textile, conductive thread and conductive yarn which could made conductive textile either in woven or knitted structures. Electrode's substrate plays an important part in developing dry and flexible ECG electrodes. Good combination (fabrication) with conductive materials produce high conductive substrate (electrode) which is able to be used for detecting bio-signal of the wearer. One of the main criteria needed for producing good quality of electrode 
is high in electrical conductivity. However, other properties (like flexibility, comfortability, and biocompatibility) [1-3] must be taken into consideration especially for wearer safety and comfortable issues.

There are several flexible substrates have been used to fabricate dry and flexible electrode for example polyethylene terephthalate (PET) [4, 5], polyurethane (PU) nanoweb [6], textile (e.g. woven cotton, nylon and polyester) [7, 8], polydimethylsiloxane (PDMS) [9] and foam [10]. Recently, research related textile-based electrodes are widely discovered due to their special characteristic such as easy to fabricate [11], ultra-thinness, light-weighted, high flexibility, stretchability, conformity and washable [1-3]. More importantly, the permeability characteristic of textile towards air and moisture will prevent any dermatology problems such as skin irritation and dermal inflammation which normally occurred when the surface plate gel $\mathrm{Ag} / \mathrm{AgCl}$ electrodes are used. The usage of gel in $\mathrm{Ag} / \mathrm{AgCl}$ electrode is to provide a good electrical conductivity and protection from motion artifacts [8]. However, this electrode is associated with several disadvantages such as inflexible [12], not practical for continuous monitoring system as the gel tends to dry gradually (need to reapply), would cause skin irritation when long term of use and could cause patient discomfort due to reapplication of the electrode's adhesive and the usage of the wet gel [8, 13].

Other than high conductivity and flexibility, the reliable of the textile electrode must be further investigated. The reliability of the electrode such as towards thermal and longevity effect should become the most important information in developing high quality electrode. Recently, several textile-based electrodes already exist on the market or as prototypes in research laboratories. However, they are not adapted yet to reliability effect especially effect in thermal and longevity of the electrode. Some of the researchers reported on the reliable and washable of the electrode. A. Ankhili et al [12] reported on washable and reliable textile electrode after 50 washing cycles for ECG monitoring. Three different types of fabric e.g. polyamide, cotton and polyester coated with PEDOT: PSS were employed and embedded into underwear fabric by sewing using conductive yarn. The ECG signals were monitored before and after washing cycles including in resting, walking and climbing stairs conditions [12]. Kaappa et al. [14] studied the electrical impedance of solid, non-breathable and breathable electrodes for ECG monitoring after 50 times of washing test. Electrode weight, surface resistance and impedance before and after repeated washing test of stainless steel, platinium, silver, silvered textile knit and conductive polymer were measured in the study [14].

This study investigated the effect of reduced Graphene Oxide (rGO)-coated cotton elctrodes with several temperatures set-up. Furthermore, the electrodes performance after fabrication of up to 100 days were determined and skin-electrode impedance of commercial ECG electrode $\mathrm{Ag} / \mathrm{AgCl}$ and textile-based electrodes were compared. In addition, the research was continued with the comparison of ECG signals acquired from $\mathrm{Ag} / \mathrm{AgCl}$ and $\mathrm{rGO}$-coated cotton electrode in term of $\mathrm{ECG}$ waveform and signal-to-noise ratio (SNR). Besides, the best position of electrodes applied on wearer was explored as the electrodes were aimed to be used for portable and wearable ECG monitoring in the future.

\section{RESEARCH METHOD}

Scoured cotton fabric in the dimension of $(2 \times 4.5) \mathrm{cm}$ was coated with graphene oxide (GO) using soaking method and reduced with ascorbic acid as the schematic diagram shows in Figure 1(a) and details mention in the previous study [15]. The electrical performance of the electrode was measured using LCR meter (BR5816 LCR meter) in the direction parallel to weft-thread direction of the cotton fabric. The topography and conductivity of the rGO-coated cotton fabric were observed using atomic force microscope, AFM (Axio Observer D1 Nanowizard3, Zeiss, Germany). The electrode was integrated with 1 layer of wax-cotton, 4 layer of cotton and attached with metallic snap as shown in Figure 1(b) [15]. Medical grade adheshive (3M $\mathrm{M}^{\mathrm{TM}} 1522$ Transparent Polyethylene Double Sided Medical Tape) was employed to patch electrode onto wearer skin. Note that for rectangle-shaped electrode, the warp direction of the fabric became the length-side while the weft direction became the width-side of the design as shown in Figure 1(c).

\subsection{Mechanical charaterization}

\subsubsection{Longevity test}

The reliability test of the rGO-coated cotton fabric was conducted to identify the suitable storage condition to store the electrode and the effect of the electrode with the humidity. The electrodes were placed in ambient condition $\left(24^{\circ} \mathrm{C}-27^{\circ} \mathrm{C}\right)$ and in a desiccator storage. The electrical performance of the samples in both conditions were monitored from day 1, 3, 5, 10, 30 and finally day 100 using LCR meter.

\subsubsection{Thermal test}

Thermal testing was focused to monitor the effect of electrical properties of the electrode toward human body temperature, $37^{\circ} \mathrm{C}$; especially when the electrode was applied on the human body. Two wires 
were connected at the perimeter of the sample using conductive epoxy in the warp direction. Initial conductance was recorded before conducting the experiment (room temperature) and the samples were places in the oven at $37^{\circ} \mathrm{C}$ for $30 \mathrm{~min}$. Then, the changes of the conductance reading were measured using LCR meter. The conductance was recorded continueosly with the $40^{\circ} \mathrm{C}, 45^{\circ} \mathrm{C}$ and $50^{\circ} \mathrm{C}$ with the temperature increament of half an hour.

\subsubsection{Skin-electrode impedance}

The skin-electrode interface impedance was inspected using electrochemical impedance spectroscopy (EIS) (Potentiostat VersaSTAT 3) with the voltage and frequency were set up at $10 \mathrm{mV}$ and $0.1 \mathrm{kHz}$ to $1 \mathrm{kHz}$, respectively. The impedance data was measured from one female subject (Age: 25 years old, BMI: 22), thus, skin-related variations like number of sweat glands and male-female physiological differences could be minimized. Three electrodes were applied on the left inside of the subject's forearm, where $\mathrm{Ag} / \mathrm{AgCl}$ electrode was applied as counter and reference electrodes, while the rGO-coated cotton electrode (rectangle-shaped, $9 \mathrm{~cm}^{2}$ ) functioned as working electrode. The subject's skin was cleaned with alcohol swab beforehand the experiment, and the electrodes distance between counterreference electrode and reference-working electrode were set to $8 \mathrm{~cm}$ and $4 \mathrm{~cm}$, respectively [16]. The impedance experiment was repeated by changing the working electrode with $\mathrm{Ag} / \mathrm{AgCl}$ electrode.

\subsection{Electrode testing}

The fabricated textile-based ECG electrode was further tested using a Sichiray BMD101 module starter kit with 2 inputs of the electrodes. The ECG signal was recorded using rectangle-shaped electrode with two difference electrode positions; e.g. horizontal as shown in Figure 1(d) and vertical, then, compared with the performance of $\mathrm{Ag} / \mathrm{AgCl}$ electrode in term of ECG signal waveform and SNR value. The ECG signals were recorded with a female volunteer A (Age: 22 years old, BMI: 16.4) in static condition. The electrodes together with portable device were patched onto subject left chest mimic Einthoven Triangle lead III position. The ECG signals and the raw data were transfered to the smartphone via Bluetooth communication channel using BMD101 application. The raw data was extracted and SNR value was calculated using MATLAB software [15].

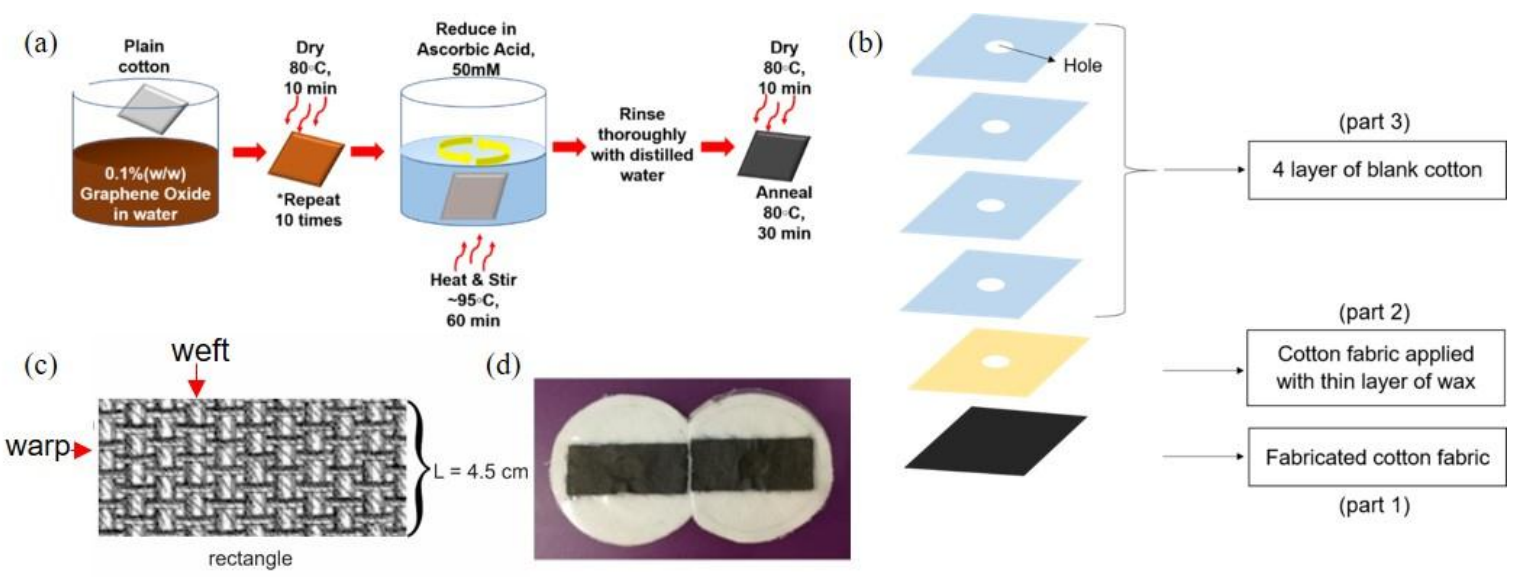

Figure 1. Fabrication of electrode, (a) Schematic illustration method of rGO-coated cotton fabric, (b) Intergration of rGO-coated cotton fabric as electrode [11], (c) The illustration image of rectangle-shaped electrode $\left(9 \mathrm{~cm}^{2}\right)$ with the indicator of weft-thread and warp-thread of cotton fabric and (d) Rectangle-shaped

(horizontal position) textile-based ECG electrode connected to Sichiray BMD101 module starter kit

\section{RESULTS AND DISCUSSION}

The electrical conductance of rectangle-shaped rGO-coated cotton electrodes are $0.79 \mathrm{mS}$ and the measurement parallel to weft-thread direction as shown in Figure 2(a). Cotton fabric was homogenously coated with GO and rGO-coated cotton fabric was successfully obtained after reduction process with ascorbic acid, proved using scanning electron microscopy (SEM) and Fourier-transform infrared spectroscopy (FTIR), respectively [15]. Material analyze of the rGO-coated cotton fabric such as surface roughness and electrical characteristic of cotton structures was further analyzed using AFM instrument. 


\subsection{Material characterization (atomic force microscopy (AFM))}

The electrical performance of rGO-coated cotton fabric was further investigated as a prove that the GO coated absorb depth into the fiber structure of the fabric. Besides, the electrical flexibility of the electrode can be investigated through the current flow at each fabric structures. Figure 2 illustrates the topography images of the rGO-coated cotton fabric from three different structures; (a) fabric, (b) thread an (c) fiber of the sample. From the characterization results, the values of current measured are decreasing from fabric to fiber structures which $11.41 \mathrm{nA}$ was observed for the fabric, $0.373 \mathrm{nA}$ and $0.338 \mathrm{nA}$ for thread and fiber, respectively. These due to the different amount of GO at each fabric structures and the position of the structures in the fabric. The results show positive indicator which it was clearly proved that the rGO absorbed until the fiber structure of the fabric. On the other hand, it reveals that the rGO-coated cotton fabric has good capability in electrical flexibility.

Furthermore, the thread illustrates high surface roughness $(1.164 \mu \mathrm{m})$ relative to the fabric and fiber structures. The fiber structure was analyzed with $414.5 \mathrm{~nm}$ and fabric with $159.4 \mathrm{~nm}$ of roughness average. These determinations due to the fabric surface was smoothly covered with thin layer of rGO and less amount of rGO on the single layer of fiber might the reason of the fiber structure has slightly highest surface roughness compared to the fabric. On the other hand, the thread structure which is made from bundle of fiber might be the main factor highest in roughness measurement. Therefore, these results proved that the GO absorb until the fiber structure due to the current reading in AFM conductivity.

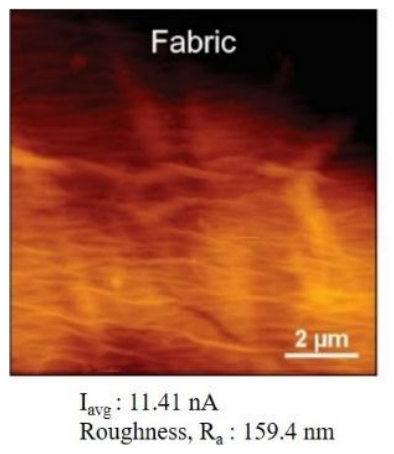

(a)
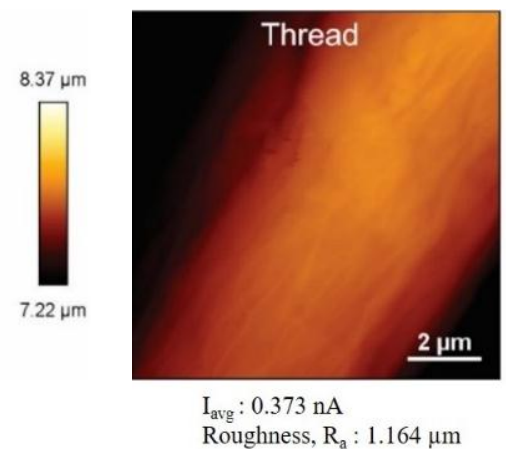

(b)
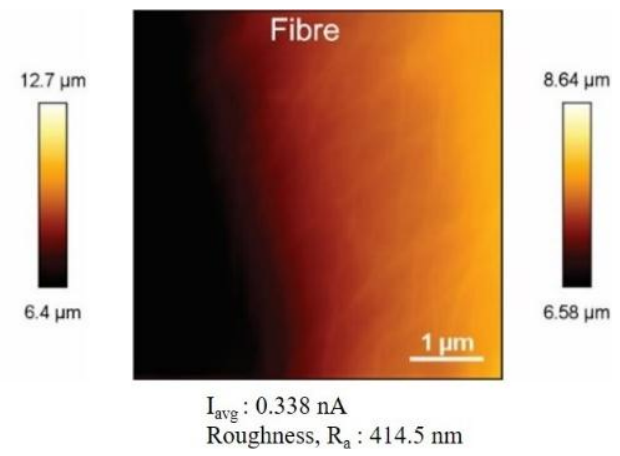

(c)

Figure 2. The topography image of, (a) Fabric, (b) Thread, (c) Fiber structures of the rGO-coated cotton fabric

\subsection{Mechanical characterization}

\subsubsection{Longevity test}

Firstly, the mechanical characterization of the fabricated cotton fabric was investigated with the longevity test. The electrical properties of rGO-coated cotton fabric with two storage conditions and the capability of the sample in maintaining its electrical properties for certain period of storage time were investigated. The rGO-coated cotton fabric was placed at two different places which are in ambient condition (room) and in the desiccator storage. The conductance changes conductance-reliability to conductance-initial $S / S_{o}$ were observed from day $1,3,5,10,30$ and 100 .

Figure 3(a) illustrates the changes of conductance for both conditions are dramatically decreased with $15.2 \%$ at day 10 and $14.3 \%$ at day 30 for desiccator and ambient storage, respectively. However, the remaining conductance for both storages maintained with slightly changes until day 100 of measurement. Results show the remaining sample's conductance that stored at ambient (room temperature) slightly higher than the sample kept in the desiccator. This might be happening due to the present of humidity in the sample that increases the conductance reading. Moreover, the samples at both storage conditions indicate the conductance remaining $>80 \%$ after 100 days of storage. This result reveals that the rGO-coated cotton fabric has a good criterion in longevity and sustainability after long period of storage time.

\subsubsection{Thermal test}

The electrode's electrical properties performance toward various temperature was examined and the main purposed of this experiment is to monitor the effect of the sample's conductance with 
the human body temperature; $37^{\circ} \mathrm{C}$, later as a function of ECG electrode. Other that than, to observe the effect of conductance with the storage and environment condition in the high temperature up to $50^{\circ} \mathrm{C}$. Figure 3(b) illustrates the details of conductance changes with various of temperature and the graph of $S / S_{o}$ versus temperature for rGO-coated cotton fabric after thermal testing, respectively. The changes of conductance with various of temperature is directly proportional to the rising of the temperature. When the specific heat was supplied, the heat is stored by the lattice vibrations (phonons) and graphene allows out-of-plane atomic displacements which known as flexural phonons [17]. Graphene is 'miracle material' with the unique structure and geometry, provided an excellent property such as thermal conductivity and thermal stability [18-21]. However, in this study the fabricated cotton fabric has some temperature limitation, due to low degradation temperature of cotton fabric which the cotton fabric will easily fragile and burn at high temperature [22]. Therefore, based on these results, the rGO-coated cotton fabric is expected to produce good signals quality when applied on human body. Other than that, the rGO-coated cotton fabric provides good thermal conductivity with the increasing of temperature and suitable for high temperature application, below $140^{\circ} \mathrm{C}$ due to limitation of cotton fabric [23].

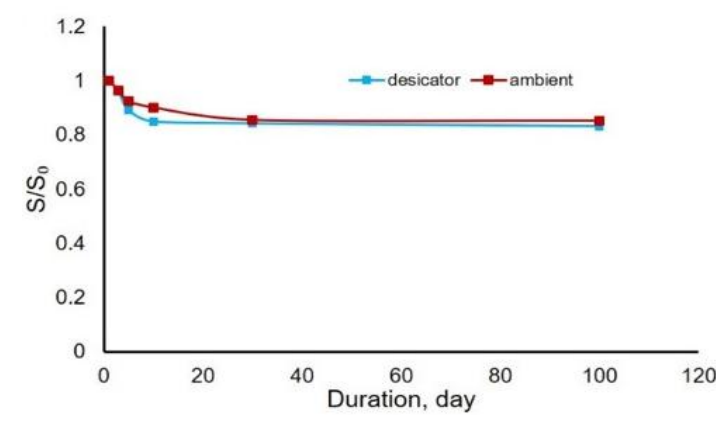

(a)

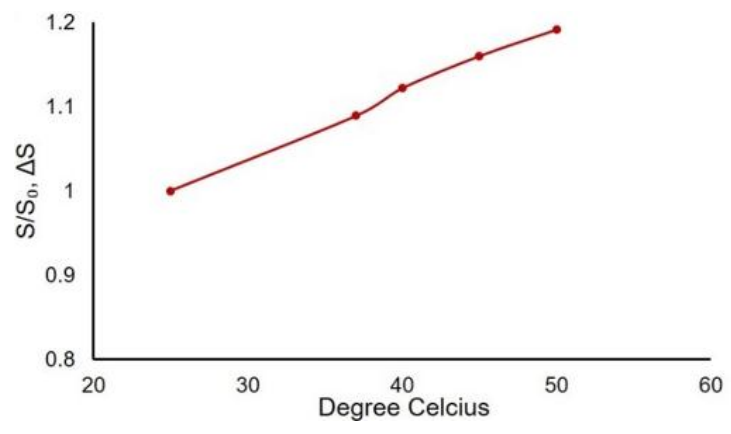

(b)

Figure 3. Mechanical characterization: the changes of sample's conductance, (a) After day 1, 3, 5, 10, 30, 100 of storage time $(\mathrm{N}=4)$, (b) With various of temperature setup, $\mathrm{N}=3$

\subsubsection{Skin-electrode impedance}

The skin-electrode interface impedance is one of the most important characterization in development ECG electrode especially for developing dry ECG electrode. It indicates that the less impedance shows the better skin-electrode contact, thus, result high quality of captured signals. Figure 4 illustrates the impedance comparison of rectangle-shaped rGO-coated cotton and $\mathrm{Ag} / \mathrm{AgCl}$ electrodes in the frequency range $0.1 \mathrm{~Hz}-1000 \mathrm{~Hz}$. The $\mathrm{Ag} / \mathrm{AgCl}$ electrode shows lower lower in skin-electrode impedance compared to rectangle-shaped rGO-coated cotton electrode with $2.93 \times 10^{5} \Omega$ and $3.91 \times 10^{5}$, respectively. This result due to the usage of gel which enhance the contact between skin and the electrode surface. However, the impedance measurement for textile-based electrode still acceptable for the dry-type electrode which previous studies revealed that the impedance can reach several hundred kilo-ohms to mega-ohms especially for textile-based and polymer-based electrode $[4,11,24]$. Note that the position of rectangle-shaped electrode at the vertical position.

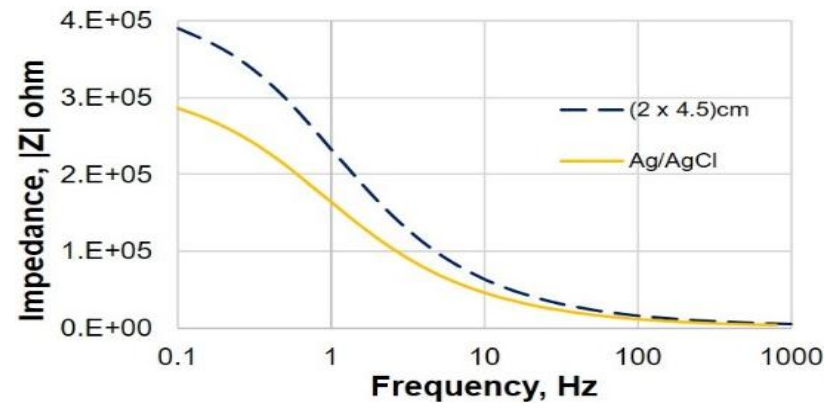

Figure 4. The skin-electrode interface impedance $(\mathrm{Z})$ of the rectangle-shaped rGO-coated cotton and $\mathrm{Ag} / \mathrm{AgCl}$ electrodes, $\mathrm{N}=6$ 


\subsection{Electrode testing}

In this testing the performance of rectangle-shaped electrode was further investigated. Figure 5 shows the ECG signals and SNR measurement of the signal acquired from $\mathrm{Ag} / \mathrm{AgCl}$ and rectangle-shaped electrodes after post-analysis using MATLAB software. From the observation, SNR value of rectangle-electrode as shown in Figure 5(a) is higher $1.09 \%$ than $\mathrm{Ag} / \mathrm{AgCl}$ as shown in Figure 5(b) electrode which the SNR value of $\mathrm{Ag} / \mathrm{AgCl}$ electrode is $16.32 \pm 0.05 \mathrm{~dB}$. For the difference position applied of rectangle-shaped electrode, it shows good ECG signals when in vertical position (same with the position in the skin-electrode impedance) however, high signal artifact, high baseline drift level and low in signal amplitude was captured in the horizontal position of the electrode as illustrates in Figure 5(c). In the horizontal position, the distance end to end between two electrodes to the metal snaps are longer compared to the vertical position of the electrodes. The electrodes position affects the quality of ECG signal acquired from the subject especially for asymmetrical shape; rectangle-shaped due to different conductivity measurement in weaving properties of the cotton fabric (warp-thread and weft-thread) $[14,25]$ and difference thread length may affect the signal reading.

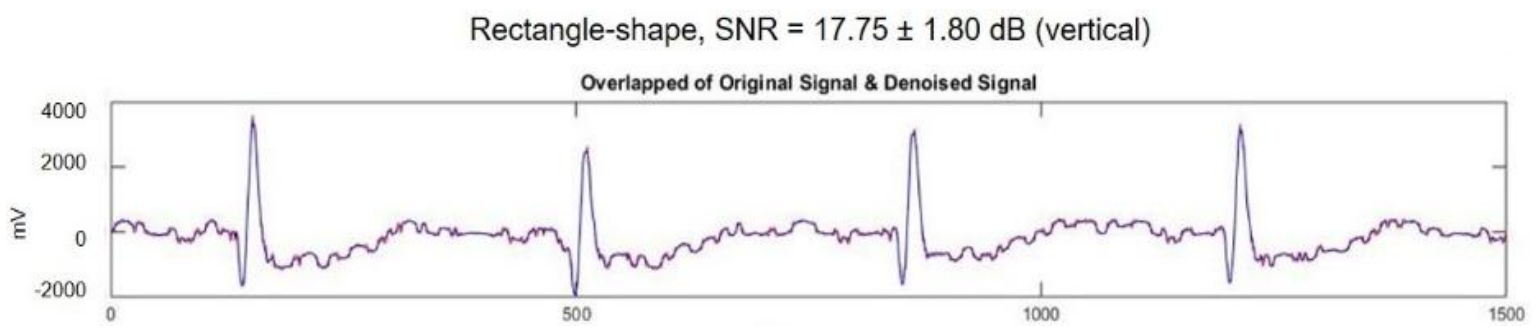

(a)

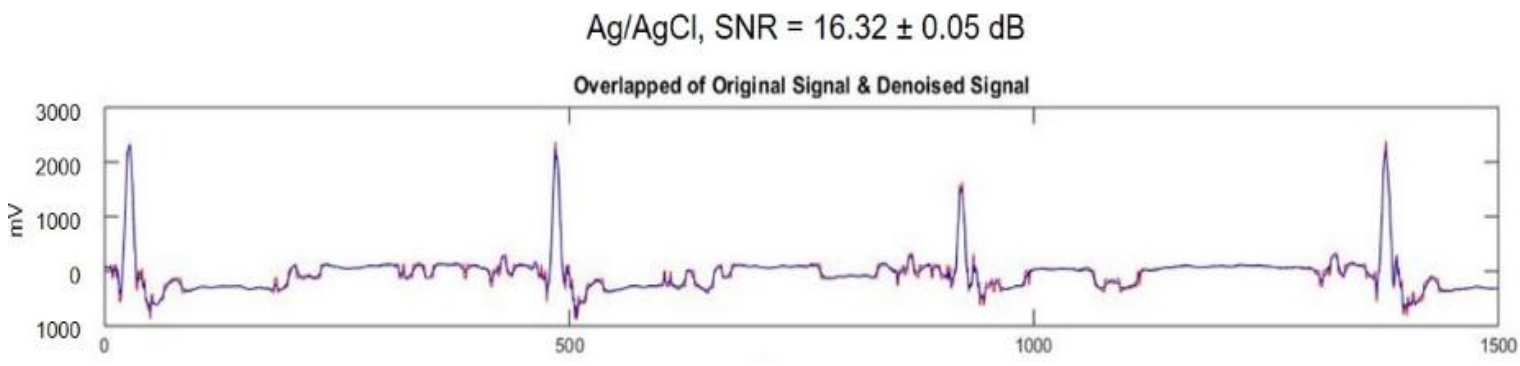

(b)

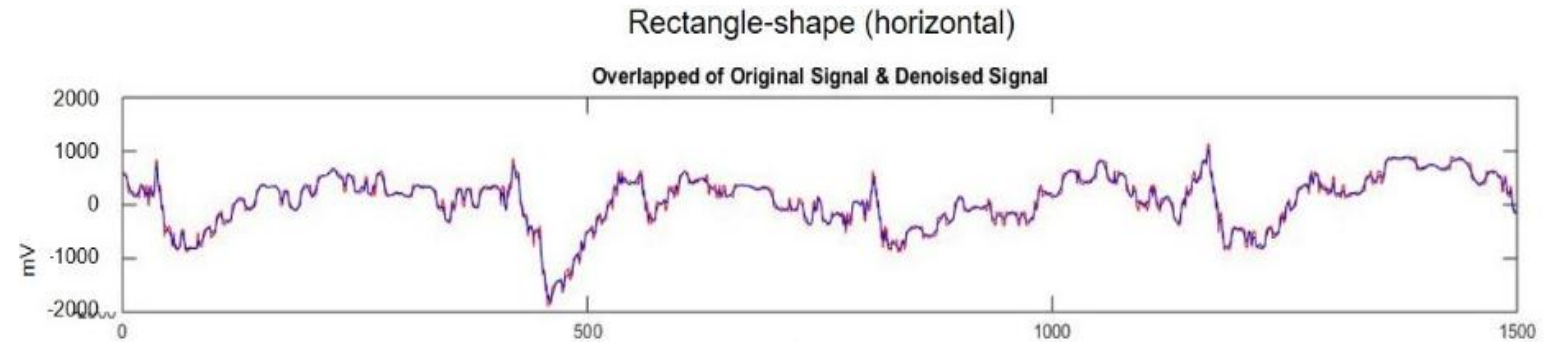

(c)

Figure 5. The overlapped signal of ECG signal together with the SNR value acquired from, (a) Rectangleshaped electrode in vertical position, (b) $\mathrm{Ag} / \mathrm{AgCl}$ electrode, (c) Rectangle-shaped electrode in horizontal position, respectively, $\mathrm{N}=6$

Table 1 shows the comparison results of rectangle-shaped rGO-coated cotton electrode with the previous research works. The rGO-coated cotton electrode with rectangle-shaped provided lower in impedance and the SNR measurement is still in range of the previous study. These results revealed that the performance of rGO-coated cotton electrode is better and comparable with the previous research work. 
Table 1 Comparison with previous research works

\begin{tabular}{ccc}
\hline Characterization & Our work & Comparison with literature \\
\hline Skin-electrode impedance $(\Omega)$ & $3.91 \times 10^{5}(1 \mathrm{~Hz}), 63.4 \mathrm{k}(10 \mathrm{~Hz})$ & $87.5 \mathrm{k}(10 \mathrm{~Hz})[11]$ \\
Signal-to-noise ratio $(\mathrm{SNR}, \mathrm{dB})$ for $\sim 4 \mathrm{~cm}$ electrode pair distance & $17.75 \pm 1.8$ & $18.4[16]$ \\
\hline
\end{tabular}

\section{CONCLUSION}

In this paper, the reliability of rGO-coated cotton fabric as ECG electrodes towards longevity and thermal had been investigated. The AFM characterization proves that the GO was absorbed to the whole structures of cotton fabric. The electrode conductance remains $>80 \%$ after 100 days of fabrication and the conductance increased with respect to the thermal supplied. The vertical position in applying the electrode for Sichiray BMD101 module starter kit device was chosen due to good signal quality. The performance of rGO-coated cotton rectangle-shaped electrode provide good or similar quality of ECG signal compared to that conventional $\mathrm{Ag} / \mathrm{AgCl}$ electrode. In a conclusion, the results of this study provide the useful information in the developing electrode and rGO-coated cotton electrode shows high potential as ECG electrode.

\section{ACKNOWLEDGEMENTS}

The authors would like to express their appreciation to Ministry of Higher Education Malaysia and Universiti Teknologi Malaysia for supporting and funding this study under Matching Grant No. Q.J130000.3001.01M13 and UTM RUG Tier 2 No. Q.J130000.2651.17J08. The authors would like to thank Hattrek and Bio-Inspired research group for their continuous support towards this research.

\section{REFERENCES}

[1] M. Sayem, A. Sadat, S. Hon Teay, H. Shahariar, P. L. Fink, and A. Albarbar, "Review on Smart Electro-Clothing Systems (SeCSs)," Sensors, vol. 20, no. 3, 2020.

[2] W. Zeng, L. Shu, Q. Li, S. Chen, F. Wang, and X. M. Tao, "Fiber- based wearable electronics: a review of materials, fabrication, devices, and applications," Advanced Materials, vol. 26, no. 31, pp. 5310-5336, 2014.

[3] C. Liao, M. Zhang, M. Y. Yao, T. Hua, L. Li, and F. Yan, "Flexible organic electronics in biology: materials and devices," Advanced materials, vol. 27, no. 46, pp. 7493-7527, 2015.

[4] A. A. Chlaihawi, B. B. Narakathu, S. Emamian, B. J. Bazuin, and M. Z. Atashbar, "Development of printed and flexible dry ECG electrodes," Sensing and Bio-Sensing Research, vol. 20, pp. 9-15, 2018.

[5] C. Lou et al., "Flexible Graphene Electrodes for Prolonged Dynamic ECG Monitoring," Sensors, vol. 16, no. 11, 2016.

[6] E. Lee and G. Cho, "PU nanoweb-based textile electrode treated with single-walled carbon nanotube/silver nanowire and its application to ECG monitoring," Smart Materials and Structures, vol. 28, no. 4, pp. 1-11, 2019.

[7] D. Pani, A. Dessì, J. F. Saenz-Cogollo, G. Barabino, B. Fraboni and A. Bonfiglio, "Fully Textile, PEDOT:PSS Based Electrodes for Wearable ECG Monitoring Systems," in IEEE Transactions on Biomedical Engineering, vol. 63, no. 3, pp. 540-549, March 2016.

[8] N. Hallfors, S. Al Junaibi, K. Liao, M. Ismail, and A. Isakovic, "Reduced Graphene Oxide for the Design of Electrocardiogram Sensors: Current Status and Perspectives," in The IoT Physical Layer: Springer, pp. 3-11, 2019.

[9] H. Jung et al., "CNT/PDMS Composite Flexible Dry Electrodesfor Long-Term ECG Monitoring," in IEEE Transactions on Biomedical Engineering, vol. 59, no. 5, pp. 1472-1479, May 2012.

[10] K. C. Tseng, B. Lin, L. Liao, Y. Wang and Y. Wang, "Development of a Wearable Mobile Electrocardiogram Monitoring System by Using Novel Dry Foam Electrodes," in IEEE Systems Journal, vol. 8, no. 3, pp. 900-906, Sep 2014.

[11] M. K. Yapici, T. Alkhidir, Y. A. Samad, and K. Liao, "Graphene-clad textile electrodes for electrocardiogram monitoring," Sensors and Actuators B: Chemical, vol. 221, pp. 1469-1474, Dec 2015.

[12] A. Ankhili, X. Tao, C. Cochrane, D. Coulon, and V. Koncar, "Washable and Reliable Textile Electrodes Embedded into Underwear Fabric for Electrocardiography (ECG) Monitoring," Materials, vol. 11, no. 2, 2018.

[13] M. Weder et al., "Embroidered Electrode with Silver/Titanium Coating for Long-Term ECG Monitoring," Sensors, vol. 15, no. 1, pp. 1750-1759, 2015.

[14] E. S. Kaappa et al., "The electrical impedance measurements of dry electrode materials for the ECG measuring after repeated washing," Research Journal of Textile and Apparel, vol. 21, no. 1, pp. 59-71, 2017.

[15] S. M. Saleh, S. M. Jusob, N. S. Sahar, N. A. Abdul-Kadir, F. K. C. Harun, and D. H. B. Wicaksono, "Fabrication of Cotton Fabric as Flexible Electrode in Electrocardiography Monitoring," Lecture Notes on Multidisciplinary Research and Application, Malaysia Technical Scientist Association, Malaysia, pp. 97-106, 2018.

[16] M. A. Yokus and J. S. Jur, "Fabric-Based Wearable Dry Electrodes for Body Surface Biopotential Recording," in IEEE Transactions on Biomedical Engineering, vol. 63, no. 2, pp. 423-430, Feb 2016.

[17] E. Pop, V. Varshney, and A. K. Roy, "Thermal properties of graphene: Fundamentals and applications," MRS Bulletin, vol. 37, no. 12, pp. 1273-1281, 2012.

[18] B. Zhang, Y. Wang, and G. Zhai, "Biomedical applications of the graphene-based materials," Materials Science and Engineering: C, vol. 61, pp. 953-964, April 2016. 
[19] A. K. Geim, “Graphene: status and prospects," Science, vol. 324, no. 5934, pp. 1530-1534, 2009.

[20] S. Syama and P. Mohanan, "Safety and biocompatibility of graphene: A new generation nanomaterial for biomedical application," International journal of biological macromolecules, vol. 86, pp. 546-555, May 2016.

[21] J. T. Robinson et al., "Ultrasmall reduced graphene oxide with high near-infrared absorbance for photothermal therapy," Journal of the American Chemical Society, vol. 133, no. 17, pp. 6825-6831, 2011.

[22] M. Shateri-Khalilabad and M. E. Yazdanshenas, "Fabricating electroconductive cotton textiles using graphene," Carbohydrate polymers, vol. 96, no. 1, pp. 190-195, July 2013.

[23] N. Karim et al., "Scalable production of graphene-based wearable e-textiles," ACS nano, vol. 11, no. 12, pp. 12266-12275, 2017.

[24] Y. M. Chi, T. Jung and G. Cauwenberghs, "Dry-Contact and Noncontact Biopotential Electrodes: Methodological Review," in IEEE Reviews in Biomedical Engineering, vol. 3, pp. 106-119, 2010.

[25] M. Tokarska, "Measuring resistance of textile materials based on Van der Pauw method," Indian Journal of Fibre \& Textile Research, vol. 38, no. 2, pp. 198-201, Jun 2013.

\section{BIOGRAPHIES OF AUTHORS}
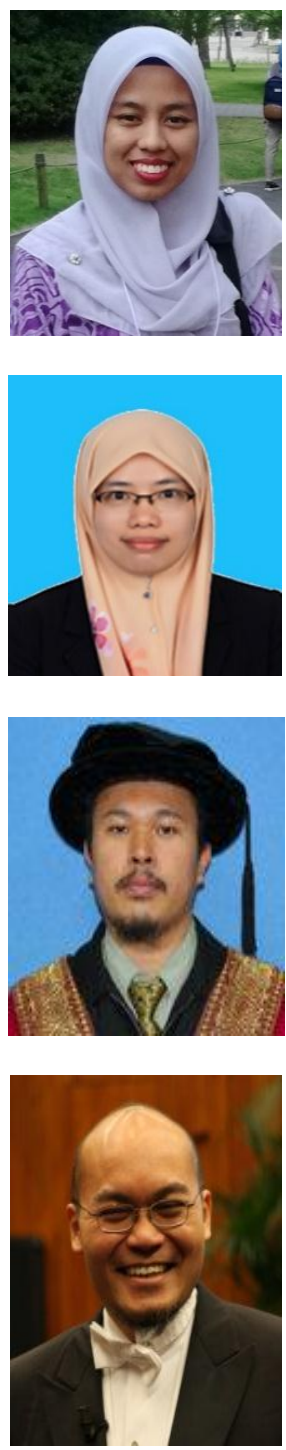

Syaidah Md Saleh received the bachelor's degree (2011) and master's degree (2014) in biomedical engineering from Universiti Teknologi Malaysia, Malaysia. She is currently a Phd's student in the Department of Biomedical Engineering in Universiti Teknologi Malaysia, Malaysia. Her research focuses on developing textile-based electrode using reduced Graphene Oxide as the conductive material and cotton fabric as a substrate for ECG applications.

Nurul Ashikin Abdul-Kadir serves at Universiti Teknologi Malaysia in the field of Electrical and Electronic Engineering as Senior Lecturer since 2019. She received her master and phD degree in Electrical Engineering and her bachelor's degree in electrical engineering (Medical Electronics). Her research interest related to electronic circuit, bio-signal processing (ECG, EEG, EMG), embedded electronics and pattern recognition.

Fauzan Khairi Che Harun received his BEng degree in Electrical-Electronics Engineering from Universiti Teknologi Malaysia (UTM) in 2003. He then further his study in the area of biomedical engineering in University of Warwick where he was awarded with MSc in Advanced Electronic (2005) and PhD in Engineering (2010). His research interests are mainly biomedical electronics, Biological Inspired Micro-system and Electronic Nose System. Currently he is a senior lecturer at the Faculty of Electrical Engineering, UTM. He also serves as the International Coordinator for Erasmus Mundus IDEAS student exchange program.

Dedy H. B. Wicaksono received his bachelor's degree in engineering (engineering physics) from Institut Teknologi Bandung, Indonesia in 1998. He then continued his study in Biological Information engineering in Tokyo Institute of Technology where he was awarded Master of Engineering in 2002. He obtained his Doctor of Philosophy in Microelectronics from Delft University of Technology in 2008. After which, he worked at the same university as Postdoctoral researcher until 2010 developing dental drill-bit integrated optical imaging and sensing. After working as lecturer at the Faculty of Biosciences and Medical Engineering, Universiti Teknologi Malaysia (UTM) from 2010 to 2016, he currently works as Assistant Professor and Head of the Department of Biomedical Engineering, Swiss German University (SGU), Indonesia. His research interest is in textile-based biomedical devices. 